\title{
Danon disease: Two patients with atrial fibrillation in a single family and review of the literature
}

\author{
SHAOHUA GUO ${ }^{1}$, LINGHUAN ZHOU ${ }^{2}$, RENPING WANG ${ }^{2}$, ZHIXIN LV $^{2}$, HONGZUN XU ${ }^{2}$, \\ BAOLI HAN $^{2}$, PANAGIOTIS KORANTZOPOULOS ${ }^{3}$, FULI HU $^{2}$ and TONG LIU ${ }^{1}$
}

\author{
${ }^{1}$ Tianjin Key Laboratory of Ionic-Molecular Function of Cardiovascular Disease, Department of Cardiology, Tianjin \\ Institute of Cardiology, The Second Hospital of Tianjin Medical University, Tianjin 300211; ${ }^{2}$ Department of Cardiology, \\ Shijiazhuang Great Wall Hospital of Integrated Traditional Chinese and Western Medicine, \\ Shijiazhuang, Hebei 050035, P.R. China; ${ }^{3}$ First Department of Cardiology, \\ University of Ioannina, Medical School, Ioannina 45110, Greece
}

Received January 19, 2019; Accepted May 31, 2019

DOI: $10.3892 /$ etm.2019.7777

\begin{abstract}
The present study reports on a family with two members affected by Danon disease but having different phenotypes. The clinical manifestations of Danon disease include cardiomyopathy, skeletal myopathy and different degrees of intellectual disability that varies greatly among patients. The present case study reports on two siblings, an older sister and a younger brother, with Danon disease from an affected pedigree, presenting with distinctly different phenotypes. The sister was diagnosed with dilated cardiomyopathy at the age of 26 years with an unfavorable outcome, while her younger brother presented with hypertrophic cardiomyopathy in a relatively stable state. The two probands shared the same mutation, c.974delTinsAA in exon 8, in the lysosomal-associated membrane protein- 2 gene. Of note, the two patients had a pre-excitation pattern in the electrocardiogram on initial presentation and later developed atrial fibrillation (AF), which markedly aggravated heart failure. To the best of our knowledge, AF has not been widely reported in patients with Danon disease. The development of AF may have a prognostic value under these circumstances.
\end{abstract}

Correspondence to: Dr Fuli Hu, Department of Cardiology, Shijiazhuang Great Wall Hospital of Integrated Traditional Chinese and Western Medicine, 84 Huanghe Avenue, Shijiazhuang, Hebei 050035, P.R. China

E-mail: hufuli1@126.com

Professor Tong Liu, Tianjin Key Laboratory of Ionic-Molecular Function of Cardiovascular Disease, Department of Cardiology, Tianjin Institute of Cardiology, The Second Hospital of Tianjin Medical University, 23 Pingjiang Road, Tianjin 300211, P.R. China

E-mail: liutongdoc@126.com

Key words: Danon disease, atrial fibrillation, cardiomyopathy, lysosomal-associated membrane protein-2 deficiency, WolffParkinson-White syndrome, pre-excitation

\section{Introduction}

Danon disease is a rare $\mathrm{X}$-linked disorder clinically characterized by a triad of cardiomyopathy, intellectual impairment and skeletal myopathy (1). Danon disease is caused by genetic defects in the lysosome-associated membrane protein 2 (LAMP2) gene, which encodes the LAMP2 protein (2). Cardiac involvement is prominent in patients with Danon disease, while left ventricular hypertrophy and dilation may be present with the former being more prevalent in males $(2,3)$. Cardiac symptoms usually occur in adolescence and during the third decade of life, while most patients die from heart failure. Electrical conduction abnormalities are also common, presenting in $>3 / 4$ of males and females with Danon disease. Specifically, ventricular pre-excitation is the most common electrocardiographic pattern (3).

Danon disease is an inherited disease that may be passed on to the next generation. The present study reports on two cases of Danon disease in a single family with distinctly different phenotypes. In this pedigree, three generations involving 6 family members were assessed, including one case of sudden death (Patient I:2), and two cases of cardiomyopathy (Patients II.1 and II.3). Patients II.1 and II.3 developed atrial fibrillation (AF) in the later course of the disease, which is not common. In addition, a literature review was performed by searching PubMed on the aspects of clinical course, phenotype-genotype association, optimal management and prognosis of Danon disease.

\section{Case report}

Patient II.1. Patient II.1 was the older child of the family, the history of which comprised sudden cardiac death in the patient's mother of 47 years old and hypertrophic cardiomyopathy (HCM) in the patient's brother (Patient II.3). The pedigree chart is provided in Fig. 1. Patient II.1, a 26-year-old female, was initially diagnosed with dilated cardiomyopathy (DCM) based on echocardiographic examination in a local hospital several months previously and then gradually developed aggravated shortness of breath and edema of the bilateral 
lower limbs. She was referred to Department of Cardiology, Shijiazhuang Great Wall Hospital of Integrated Traditional Chinese and Western Medicine in March 2016. On physical examination, the patient was hemodynamically stable, with positive hepatojugular reflex and edema in the lower limbs. Pertinent laboratory parameters included elevated lactate dehydrogenase (344.09 U/1; normal range, 120-250 U/1), elevated $\alpha$-hydroxybutyrate dehydrogenase (388.96 U; normal range, 72-182 U/l), elevated $\mathrm{N}$-terminal pro-brain natriuretic peptide $(4,212 \mathrm{pg} / \mathrm{ml}$; normal range, $0-450 \mathrm{pg} / \mathrm{ml})$, elevated aspartate transferase (49.81 U/l; normal range, 13-35 U/l) and elevated $\gamma$-glutamyltranspeptidase (135.14 U/1; normal range, 7-45 U/l). The electrocardiogram (ECG) exhibited a ventricular pre-excitation pattern at the first hospitalization (Fig. 2A). At nine months later, the patient was admitted due to advanced heart failure with persistent AF (Fig. 2B). The echocardiographic examination revealed mild left ventricular hypertrophy (interventricular septum, $13 \mathrm{~mm}$; left ventricle anterior wall, $12 \mathrm{~mm}$ ) and dilated left heart (atrium, $37 \mathrm{~mm}$; left ventricle at end diastole, $63 \mathrm{~mm}$ ) (Fig. 2C). The left ventricular systolic function was severely impaired with an ejection fraction of 29\% (Fig. 2D).

Biopsy specimens were taken from the patient's left deltoid muscle. NADH enzyme staining indicated that small irregular vacuoles were scattered in certain muscle fibers, and a small amount of type I muscle fiber aggregated in edge particles (Fig. 3C). ATPase staining revealed clustering in some areas (Fig. 3B). The pathological diagnosis was myogenic fiber disorder myopathy (Fig. 3).

For sequence analysis, DNA was extracted from blood. A heterozygous frameshift missing mutation was identified in the LAMP2 gene of the patient. The proband harbored an identical c.974delTinsAA in exon 8 (Fig. 2E).

Collectively, the patient was admitted to the hospital 6 times for heart failure decompensation within 2 years and experienced sudden cardiac death during her last hospitalization at the age of 28 years.

Patient II.3. Patient II.3, the younger brother of Patient II.1, was a 23 -year-old male presenting with a distinctly different clinical course and initial manifestation. He was initially referred to a local hospital due to an unexplained elevation of cardiac enzyme in April 2016, one year before his referring to our department, and dyspnea and weakness followed. Clinical examination revealed hepatomegaly with no obvious edema. He reported mild fatigue during physical activity. Abnormalities in laboratory parameters included serum procarbamide transaminase (155 U/1; normal range, 9-50 U/l), serum aspartate aminotransferase (215 U/l; normal range, 15-40 U/l), serum creatine kinase (945 U/1; normal range, 24-194 U/1), serum creatine kinase isoenzyme (45.40 U/1; normal range, 0-24 U/l), serum $\alpha$-hydroxybutyrate dehydrogenase (711 U/l; normal range, 24-194 U/l) and serum $\gamma$-glutamyl transpeptidase (72 U/1; normal range, 10-60 U/1). The chest X-ray revealed mild cardiomegaly. A ventricular pre-excitation pattern was evident in the ECG (Fig. 4A). The ECG examination revealed interventricular septum hypertrophy of $15 \mathrm{~mm}$ and diffuse thickening of the left ventricular wall (Fig. 4C). The patient had a decreased left ventricular systolic function with an ejection fraction of $37 \%$ (Fig. 4D). Pathological diagnosis was also
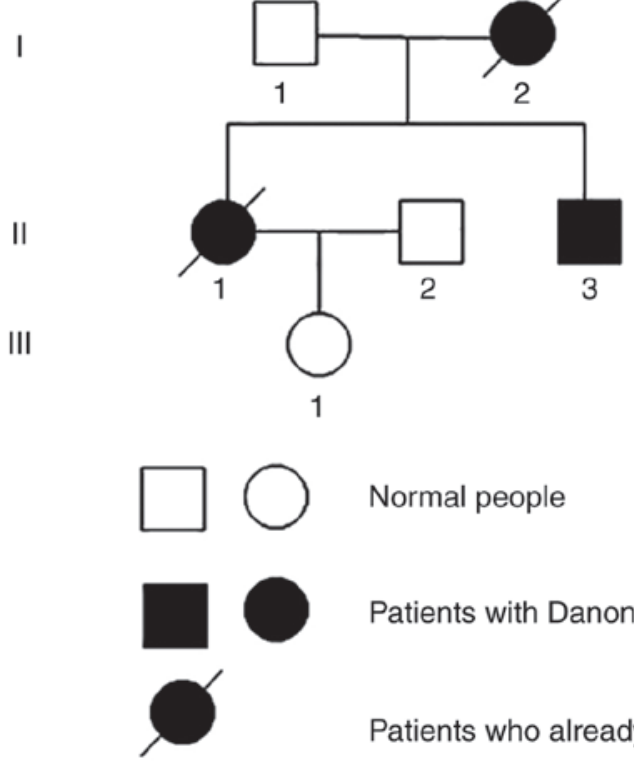

Normal people

Patients with Danon disease

Patients who already died

Figure 1. Pedigree chart of the family with Danon disease.

myogenic fiber disorder myopathy, similar to that in patient II.1 but with more obvious vacuoles in fibers.

DNA sequence analysis was also performed using a sample extracted from an oral swab. An identical mutation to that in patient II.1 was detected; the only difference was in the homozygous presentation.

Over one year, patient II.3 was admitted to the hospital 3 times for heart failure decompensation, while pertinent laboratory results and cardiac function estimated by ECG did not indicate any significant alterations. The patient developed AF (Fig. 4B) during his second hospitalization and $\sim 1$ year later his sinus rhythm was restored following catheter radiofrequency ablation. At the time of writing of the manuscript, one year after the intervention, the patient remained in a relatively stable state.

Other family members.Patients I.1 and III.1 had normal psychomotor development and no exercise intolerance. The LAMP2 gene mutation was not identified in these two family members. Patient I.2, the mother of Patient II.1, suffered a sudden cardiac death without an accurate diagnosis. Patient II.2 was normal with no sign of mental or physical disorders.

\section{Discussion}

The present study described 2 cases of Danon disease in a single family, which clearly differed in terms of severity of the underlying cardiomyopathy. In previous cases, AF has not been widely described to develop after progression of the disease, as in the two patients of the present study. In the subsequent section, a review of the literature is provided and the epidemiology, manifestations, cardiac electrical abnormalities, imaging examinations and genetics of Danon disease are discussed.

The literature search was performed using PubMed to identify articles and case reports without any time limitation to identify articles and case reports on Danon disease. The search terms were a combination of 'Danon disease', 'lysosomal glycogen storage disease without acid maltase deficiency' 
A
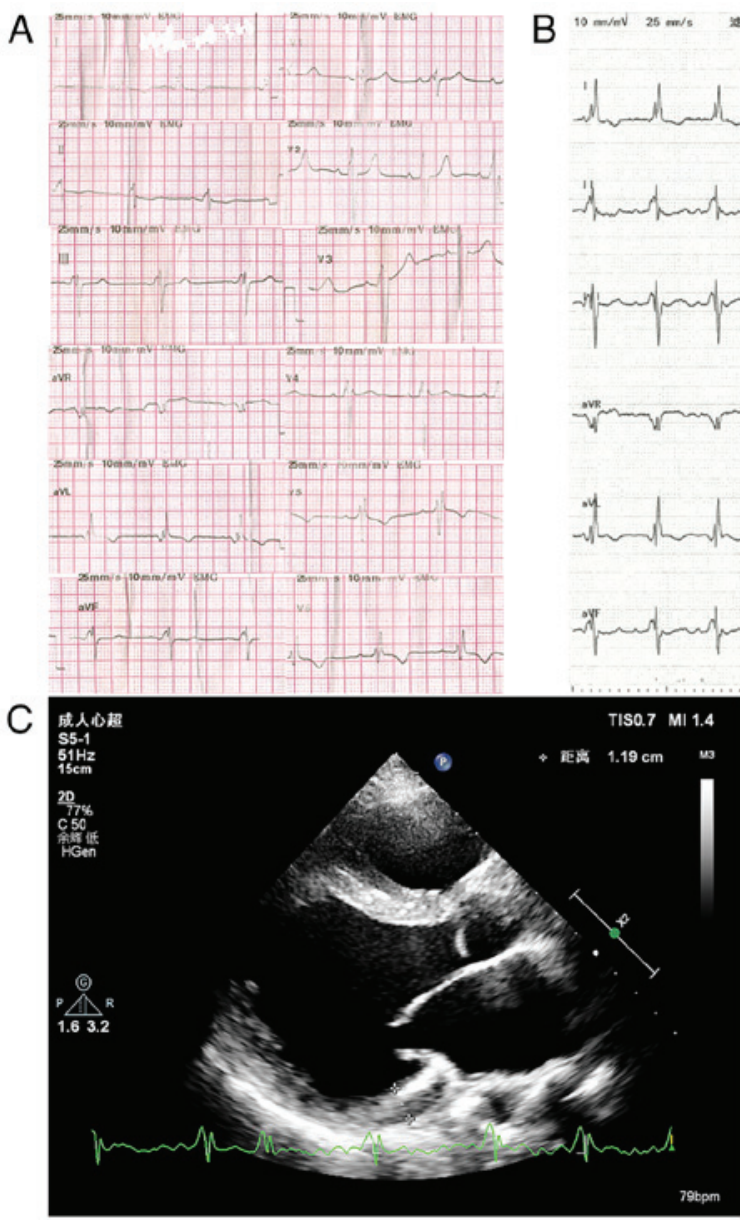

$\mathrm{B}$
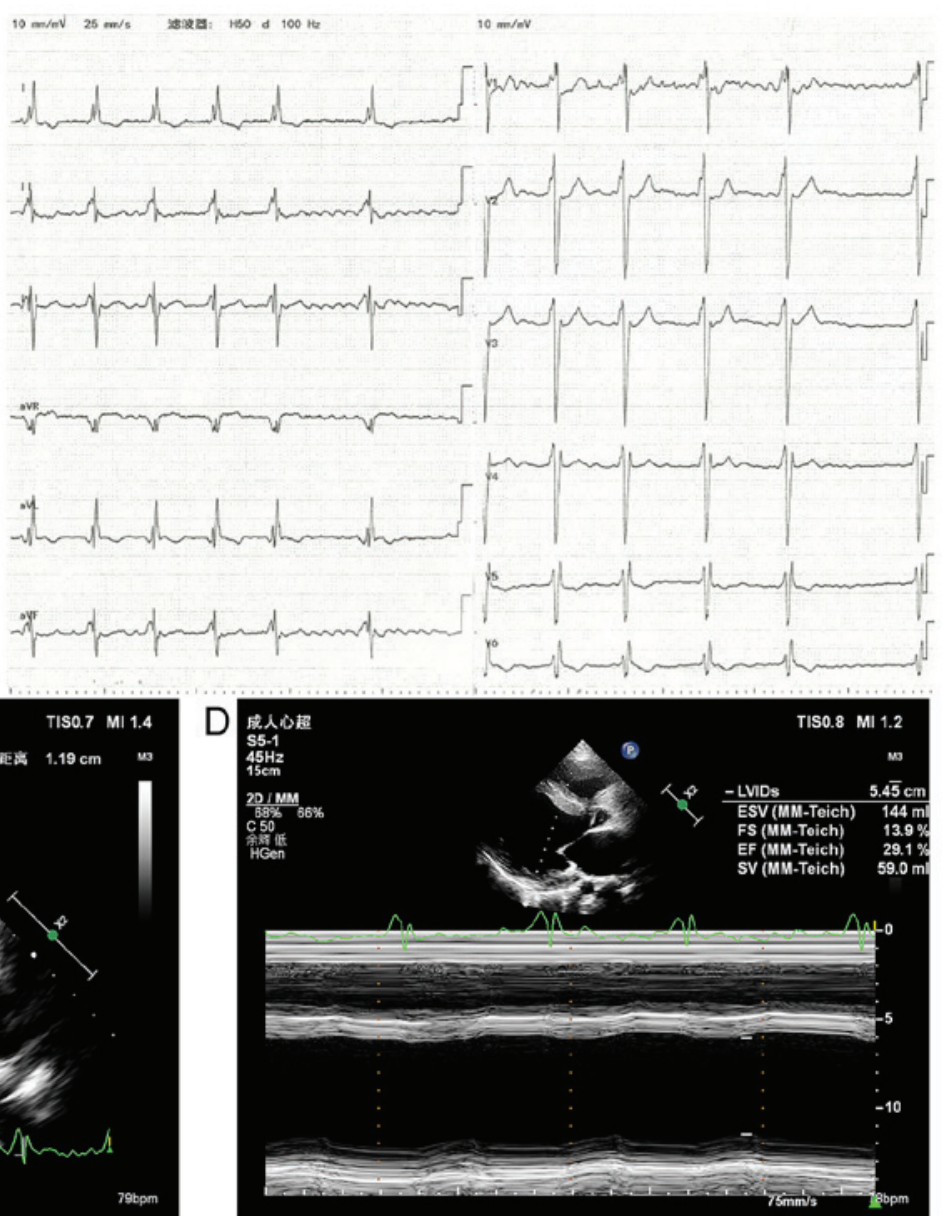

$\mathrm{E}$

Detection site: LAMP2 c.974del T P.Leu325Argfs*21 (rs730880498)

Control sequence A T A A G A A C T T C C C A G G G G G G C A T C C C A G T A G C

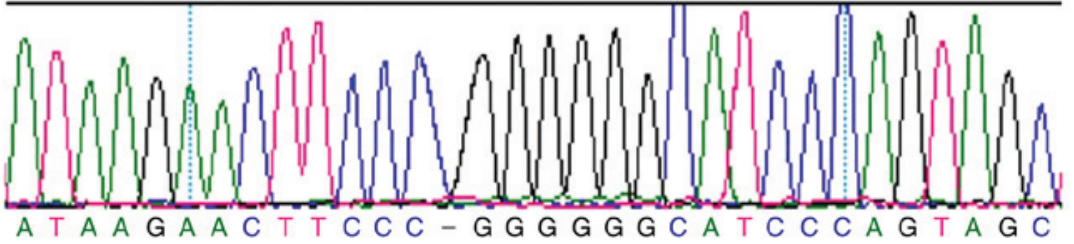

Sample sequence

Figure 2. (A) ECG at the first hospitalization indicated a Wolff-Parkinson-White pre-excitation pattern. (B) ECG recorded nine months later exhibited an atrial fibrillation pattern. (C and D) M-mode tracing in left parasternal long-axis echocardiogram indicated a dilated left ventricle. P showed the direction of ultrasonic probe, X2 indicated a magnification of two times. (E) DNA sequence chromatogram of the LAMP2 c.974delTinsAA mutation detected in the patient. LAMP2, lysosomal-associated membrane protein-2; ECG, electrocardiogram. ESV, left ventricular end-systolic volume; FS, left ventricular shortening rate; $\mathrm{EF}$, left ventricular ejection fraction; SV, left ventricular output per stroke.

and 'glycogen storage disease IIb'. First, the epidemiology of Danon disease was reviewed. Of note, the majority of published data on Danon disease originate from case reports and the prevalence of this disease is undetermined. A recent study reported a prevalence of $1.5 \%$ in a cohort with unexplained left ventricular hypertrophy, rising to $30 \%$ in patients with unexplained left ventricular hypertrophy and pre-excitation on ECG (4). Almost all patients had the manifestation of cardiomyopathy, with males predominantly presenting with a HCM phenotype, while females presented with HCM or DCM equally (3). It is noteworthy that patients with idiopathic HCM most commonly exhibit preserved systolic function and better prognosis (5). However, patients with Danon disease presenting with an HCM pattern and manifesting with preserved systolic function early in the course of disease may later progress with a worse prognosis. One study identified LAMP2 mutations in 4 of 64 patients with HCM, while no LAMP2 mutation was detected in 72 patients with DCM (6). In another study of an affected family, contrary to common sense, DCM was predominant in male patients (7). Of note, the prevalence of diagnosed cases of Danon disease may be rising due to increased detection based on the expanding knowledge of this rare disease.

The clinical features of Danon disease were then reviewed. Danon disease has been increasingly reported, mostly as case reports, during the past few years. To the best of our knowledge, female patients with Danon disease mostly present with mild symptoms and a later onset $(13.3 \pm 8.0$ years of age for male patients and $28.9 \pm 14.2$ years for female patients; $\mathrm{P}=0.0008$ ) (3). Of note, the female patient of the 

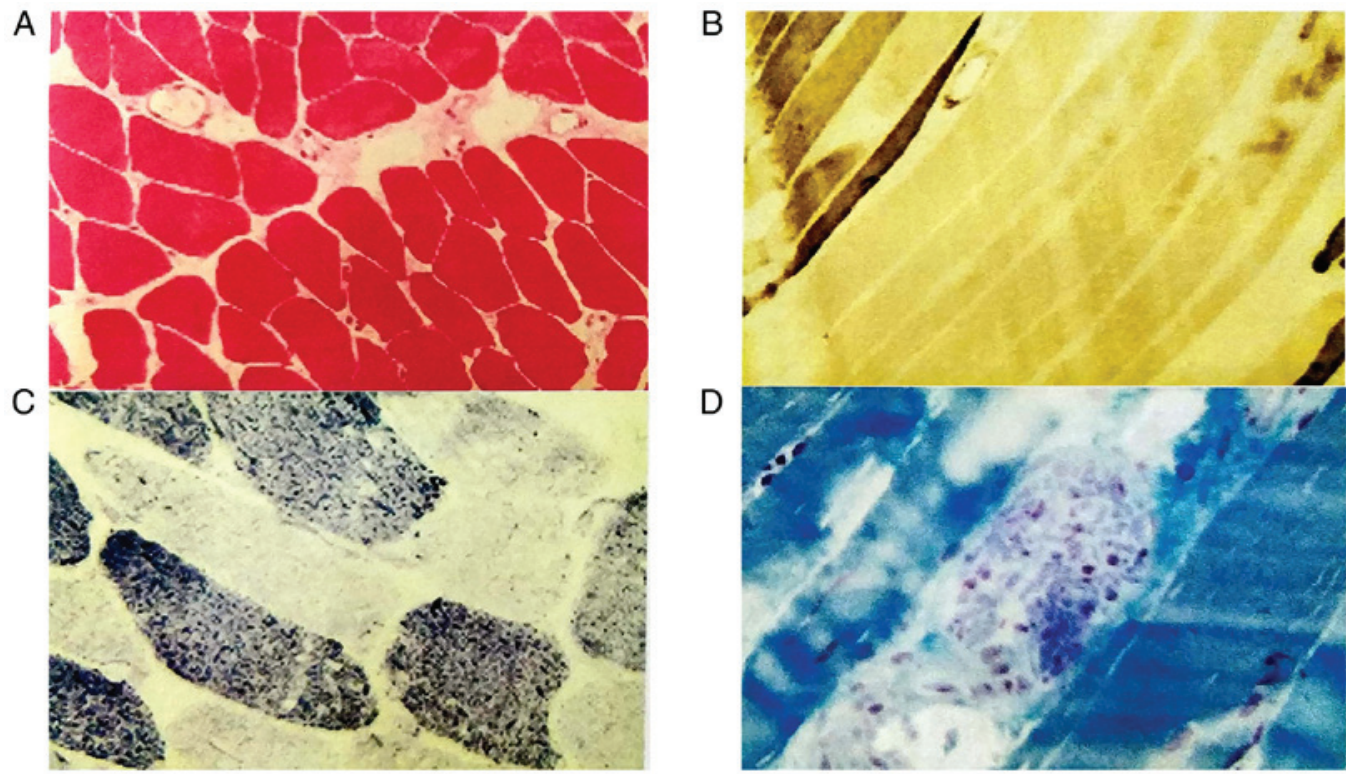

Figure 3. Light microscopy images of left deltoid muscle specimens. (A) Histochemical analysis with HE staining: Muscle fibers of mild atrophy are irregular. (B) ATPase staining indicates clustering in certain areas. (C) NADH enzyme staining reveals small irregular cavities scattered in certain muscle fibers and few particles are slightly accumulated around a small amount of I-type muscle fiber edges. (D) Modified Gomori staining: No significant decrease in the number of nerve fibers (compared with normal amounts) between the muscle fibers was noted. HE, hematoxylin-eosin; NADH, nicotinamide adenine dinucleotide; ATPase, adenosine triphosphatase. Magnification for A: x200, magnification for B-D: x400.

A
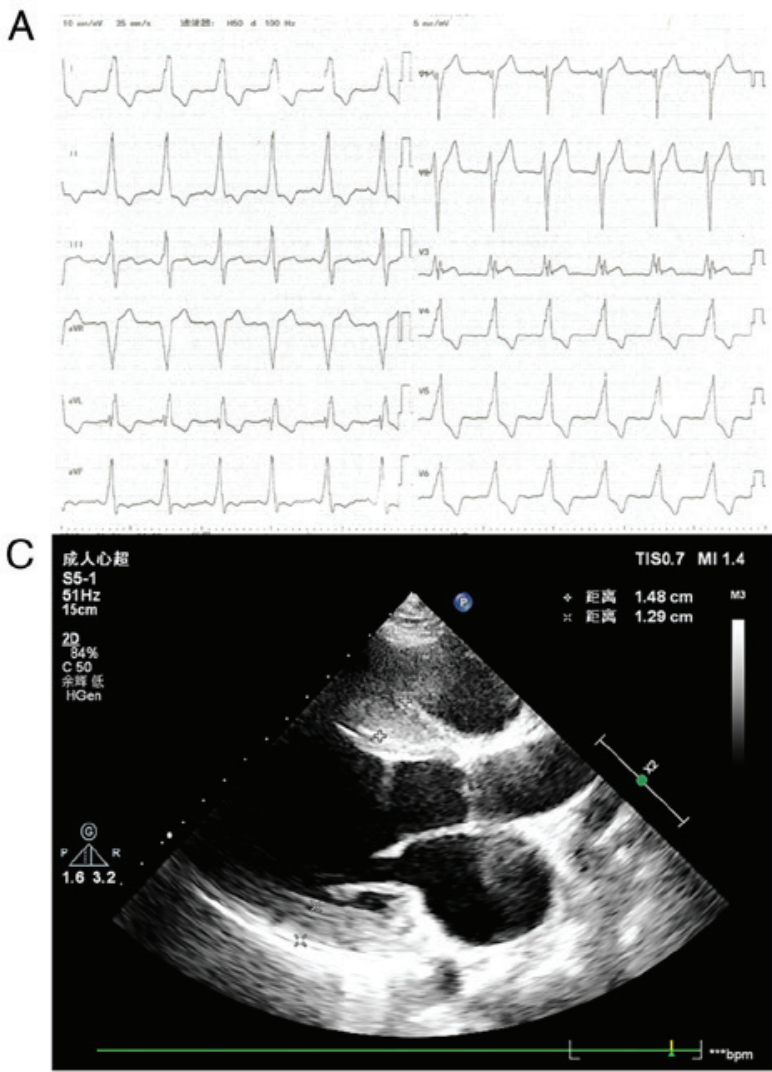

B
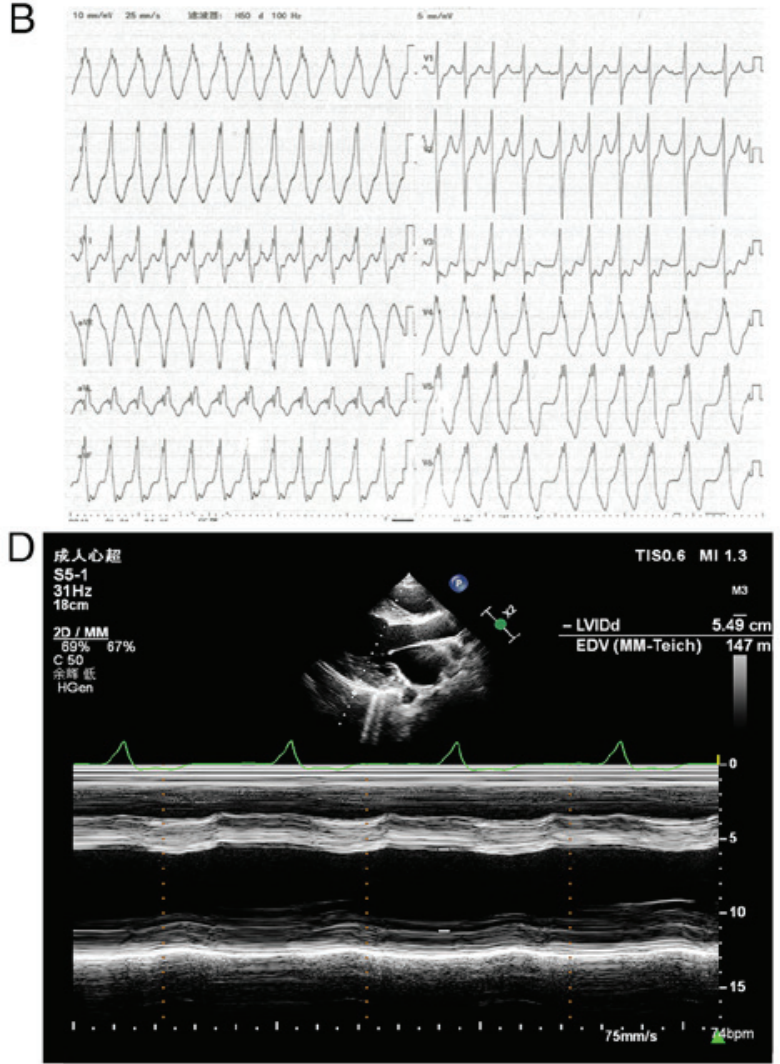

Figure 4. (A) Electrocardiogram at the first hospitalization featuring a Wolff-Parkinson-White pre-excitation pattern. (B) Electrocardiogram at the second hospitalization with atrial fibrillation pattern. (C and D) M-mode tracing in left parasternal long-axis echocardiogram exhibited left ventricular hypertrophy and decreased left ventricular ejection fraction. P shows the direction of ultrasonic probe, X2 indicates a magnification of two times. EDV, left ventricular end diastolic volume.

present study manifested with an early onset as a female and had an unfavorable prognosis. Indeed, the phenotypes in the different genders are distinctly different given that males are homozygous and females are heterozygous, presumably due to the gene dosage difference with haploinsufficiency and skewed X-chromosome inactivation (8-10). The clinical 
course in female patients with Danon disease may have a broad spectrum. The youngest female patient reported had an onset at the age of 12 years (11). In addition, a 38-year-old female, whose son was diagnosed with Danon disease based on genetic analysis, harbored the same mutation as her son and was asymptomatic with no specific abnormalities in the ECG, physical examination and blood biochemistry (12). Regarding the variety and severity of cardiomyopathy symptoms, Hedberg Oldfors et al (13) reported that an uneven distribution of LAMP2 protein causing deleterious effects in the myocardium may have a more significant role than an overall moderate reduction of LAMP2 protein. Cardiomyopathy is present in almost all patients with Danon disease. Of note, the two probands of the present study did not exhibit any clinical indication of skeletal myopathy or intellectual disability. Due to lack or decrease of LAMP2 protein in all body parts of the patients, certain less prevalent symptoms may also occur. In the eye, LAMP2 is expressed only in the retinal pigment epithelium (14), and retinal abnormalities are estimated to occur in $64-69 \%$ of patients (3). Hepatic disease has also been described in patients with Danon disease $(2,15)$. Autism associated with Danon disease was reported in a 16-month-old male with normal presentation on cardiac evaluation (16). All available evidence indicates that Danon disease is a multi-system disease and therefore, attention should be paid to symptoms of systems other than cardiovascular system in order to avoid a missed diagnosis of this lethal disease. Regarding cardiac electrical abnormalities, a ventricular pre-excitation pattern was evident in the patients, but no electrophysiological evaluation was performed. Ventricular pre-excitation is the most frequent abnormality on ECG in this setting and it has been demonstrated in $68 \%$ of males and $27 \%$ of females (3). However, in a recent study, pre-excitation was identified in only 11 out of 27 patients with Danon disease (17). Of note, in a study that performed an electrophysiologic evaluation of 4 male patients with Danon disease and initial QRS complex mimicking ventricular pre-excitation on ECG, no accessory pathway was identified (18). On the other hand, a fasciculoventricular pathway was discovered on electrophysiology examination in a 13 -year-old male with Danon disease from Spain in 2008 (19). These results were consistent with those of a further case (20). In addition, a recently published study demonstrated that a fasciculoventricular pathway was present in 2 out of 3 patients with Danon disease, with no electrophysiology results available in the one remaining patient (4). According to the aforementioned studies focusing on pre-excitation, a fasciculoventricular pathway may represent a hallmark of Danon disease.

The present study provided electrocardiographic evidence of AF in the Patient II.1 and Patient II. 3 during the progression of the disease. At present, available data regarding AF in Danon disease are limited. D'Souza et al (20) reported on a 14-year-old male diagnosed with Danon disease who presented with persistent palpitations at the age of 8 years. He had Wolff-Parkinson-White syndrome, brief runs of atrial tachycardia and pre-excited AF, and experienced sudden cardiac death at the age of 14 . Konrad et al (18) observed AF in 5 out of 7 patients with Danon disease from 3 families and hypothesized that AF may be associated with thromboembolic events in young individuals, which is supported by another three cases reported previously (21). In the patients of the present study, AF developed and aggravated the heart failure. There are only few reports of AF in Danon disease, and it may indicate a worse prognosis of this disease. Therefore, it is important to pay attention to the development of AF in patients when the diagnosis is already certain. However, whether aggressive treatment of AF in this setting favorably affects the evolution of heart failure remains elusive. Of note, AF ablation in Patient II.3 of the present study effectively restored the sinus rhythm and improved his general condition.

The genetics associated with Danon disease were then reviewed. The LAMP2 c.974delT mutation identified in the cases of the present study is a frameshift mutation in exon 8 of the LAMP2 gene that was first reported by Nishino et al (1). Genotype-phenotype associations appear to have a significant role in age of onset of Danon disease. Nonsense, frameshift and large deletion/duplication mutations were associated with the earliest age of onset (22). In the family described in the present study, the mother of Patient II.1 suffered sudden death at the age of 47, respectively, much later than Patient II.1, and not in accordance with the early-onset pattern of a frameshift mutation. Bertini et al (23) reported that the same mutation of the LAMP2 gene resulted in various phenotypes. Although the mutation type significantly affected the phenotype, there are other factors contributing to the final phenotype, which may explain for the heterogeneity of phenotypes. Danon disease is caused by mutation of the LAMP2 gene; however, in a previous study, a patient with normal DNA sequencing results presented with symptoms of Danon disease, and a LAMP2 microduplication was confirmed by PCR and RT-PCR analyses (24). From the above, it is apparent that genotype-phenotype associations summed up previously may not apply in numerous cases.

Regarding imaging examination and treatment, ECG, particularly three-dimensional speckle-tracking imaging (25), and cardiac magnetic resonance (26) have a significant role in the diagnosis and assessment of Danon disease, as reported in numerous cases $(25,27)$. The final treatment of Danon cardiomyopathy is heart transplantation, while applying a left ventricular assist device at the appropriate time is a crucial management method prior to heart transplantation (28). Implantation of a cardioverter defibrillator is also a useful treatment, and subcutaneous implantable cardioverter defibrillators have the advantage of terminating ventricular tachycardia or fibrillation much better than transvenous implantable cardioverter defibrillators (29).

In conclusion, the present study reported on a frameshift mutation in the LAMP2 gene in 2 siblings with cardiomyopathy. For patients with DCM or HCM, elevated serum myocardial enzyme and serum liver enzyme, a pre-excitation in the ECG, fibrosis or dynamic abnormities on ECG examination or cardiac magnetic resonance imaging, retinopathy myopathy and intellectual impairment, particularly with an X-linked family history, point to the diagnosis of Danon disease. Muscle biopsy and genetic mutation analysis are necessary to make a definite diagnosis. The development of AF may represent a significant feature of Danon disease. The prognostic significance of AF and its impact on the management of patients with Danon disease requires further study. 


\section{Acknowledgements}

Not applicable.

\section{Funding}

No funding was received.

\section{Availability of data and materials}

The datasets used and/or analyzed during the present study are available from the corresponding author on reasonable request.

\section{Authors' contributions}

TL, FH, SG and LZ contributed to the writing and preparation of the manuscript. RW, ZL, HX and $\mathrm{BH}$ collected the clinical and imaging data. SG, LZ, RW and PK performed the literature review. SG, LZ, FH and TL were major contributors in writing and revising the manuscript. All authors read and approved the final version of the manuscript.

\section{Ethics approval and consent to participate}

Not applicable.

\section{Patient consent for publication}

The patients provided written informed consent; however, the authors made efforts to remove identifying information to protect the privacy of the patients.

\section{Competing interests}

The authors declare that they have no competing interests.

\section{References}

1. Nishino I, Fu J, Tanji K, Yamada T, Shimojo S, Koori T, Mora M, Riggs JE, Oh SJ, Koga Y, et al: Primary LAMP-2 deficiency causes X-linked vacuolar cardiomyopathy and myopathy (Danon disease). Nature 406: 906-910, 2000.

2. Sugie K, Yamamoto A, Murayama K, Oh SJ, Takahashi M, Mora M, Riggs JE, Colomer J, Iturriaga C, Meloni A, et al: Clinicopathological features of genetically confirmed danon disease. Neurology 58: 1773-1778, 2002.

3. Boucek D, Jirikowic J and Taylor M: Natural history of Danon disease. Genet Med 13: 563-568, 2011.

4. Liu Y, Wang F, Chen X, Liang Y, Deng H, Liao H, Rao F, Wei W, Zhang Q, Zhang B, et al: Fasciculoventricular pathways responsible for ventricular preexcitation in patients with danon disease. Circ Arrhythm Electrophysiol 11: e006704, 2018.

5. Rowin EJ, Maron BJ, Kiernan MS, Casey SA, Feldman DS, Hryniewicz KM, Chan RH, Harris KM, Udelson JE, DeNofrio D, et al: Advanced heart failure with preserved systolic function in nonobstructive hypertrophic cardiomyopathy: Under-recognized subset of candidates for heart transplant. Circ Heart Fail 7: 967-975, 2014.

6. Fu L, Luo S, Cai S, Hong W, Guo Y, Wu J, Liu T, Zhao C, Li F, Huang $\mathrm{H}$, et al: Identification of LAMP2 mutations in early-onset danon disease with hypertrophic cardiomyopathy by targeted next-generation sequencing. Am J Cardiol 118: 888-894, 2016.

7. Taylor MR, Ku L, Slavov D, Cavanaugh J, Boucek M, Zhu X, Graw S, Carniel E, Barnes C, Quan D, et al: Danon disease presenting with dilated cardiomyopathy and a complex phenotype. J Hum Genet 52: 830-835, 2007.
8. Dougu N, Joho S, Shan L, Shida T, Matsuki A, Uese K, Hirono K, Ichida F, Tanaka K, Nishino I and Inoue H: Novel LAMP-2 mutation in a family with Danon disease presenting with hypertrophic cardiomyopathy. Circ J 73: 376-380, 2009.

9. Arad M, Maron BJ, Gorham JM, Johnson WH, Saul JP Perez-Atayde AR, Spirito P, Wright GB, Kanter RJ, Seidman CE and Seidman JG: Glycogen storage diseases presenting as hypertrophic cardiomyopathy. N Engl J Med 352: 362-372, 2005.

10. Majer F, Vlaskova H, Krol L, Kalina T, Kubanek M, Stolnaya L, Dvorakova L, Elleder M and Sikora J: Danon disease: A focus on processing of the novel LAMP2 mutation and comments on the beneficial use of peripheral white blood cells in the diagnosis of LAMP2 deficiency. Gene 498: 183-195, 2012.

11. Sugie K, Yoshizawa H, Onoue K, Nakanishi Y, Eura N, Ogawa M, Nakano T, Sakaguchi Y, Hayashi YK, Kishimoto T, et al: Early onset of cardiomyopathy and intellectual disability in a girl with Danon disease associated with a de novo novel mutation of the LAMP2 gene. Neuropathology 36: 561-565, 2016.

12. Chen XL, Zhao Y, Ke HP, Liu WT, Du ZF and Zhang XN: Detection of somatic and germline mosaicism for the LAMP2 gene mutation c.808dupG in a Chinese family with danon disease. Gene 507: 174-176, 2012.

13. Hedberg Oldfors C, Mathe G, Thomson K, Tulinius M, Karason K, Östman-Smith I and Oldfors A: Early onset cardiomyopathy in females with Danon disease. Neuromuscul Disord 25: 493-501, 2015.

14. Schorderet DF, CottetS,Lobrinus JA, BorruatFX,Balmer A and Munier FL: Retinopathy in Danon disease. Arch Ophthalmol 125: 231-236, 2007.

15. Danon MJ, Oh SJ, DiMauro S, Manaligod JR, Eastwood A, Naidu S and Schliselfeld LH: Lysosomal glycogen storage disease with normal acid maltase. Neurology 31: 51-57, 1981.

16. Burusnukul P, de Los Reyes EC, Yinger J and Boué DR: Danon disease: An unusual presentation of autism. Pediatr Neurol 39: 52-54, 2008.

17. López-Sainz A, Salazar-Mendiguchía J, Garcia-Alvarez A, Campuzano Larrea O, Lopez-Garrido MÁ, Garcia-Guereta L, Fuentes Cañamero ME, Climent Payá V, Pena-Peña ML, Zorio-Grima E, et al: Clinical findings and prognosis of danon disease. An analysis of the Spanish multicenter danon Registry. Rev Esp Cardiol (English, Spanish) 72: 479-486, 2018.

18. Konrad T, Sonnenschein S, Schmidt FP, Mollnau H, Bock K, Ocete BQ, Münzel T, Theis C and Rostock T: Cardiac arrhythmias in patients with danon disease. Europace 19: 1204-1210, 2017.

19. García Seara FJ, Martínez Sande JL, Cid Alvarez B and González Juanatey JR: Wolff-Parkinson-White's syndrome and danon's disease. Med Clin (Barc) (Spanish) 130: 277, 2013.

20. D'Souza RS, Mestroni L and Taylor MRG: Danon disease for the cardiologist: Case report and review of the literature. J Community Hosp Intern Med Perspect 7: 107-114, 2017.

21. Spinazzi M, Fanin M, Melacini P, Nascimbeni AC and Angelini C: Cardioembolic stroke in danon disease. Clin Genet 73: 388-390, 2008.

22. D'Souza RS, Levandowski C, Slavov D, Graw SL, Allen LA, Adler E, Mestroni L and Taylor MR: Danon disease: Clinical features, evaluation, and management. Circ Heart Fail 7: 843-849, 2014

23. Bertini E, Donati MA, Broda P, Cassandrini D, Petrini S, Dionisi-Vici C, Ballerini L, Boldrini R, D'Amico A, Pasquini E, et al: Phenotypic heterogeneity in two unrelated Danon patients associated with the same LAMP-2 gene mutation. Neuropediatrics 36: 309-313, 2005.

24. Lines MA, Hewson S, Halliday W, Sabatini PJ, Stockley T, Dipch AI, Bowdin S and Siriwardena K: Danon disease due to a novel LAMP2 microduplication. JIMD Rep 14: 11-16, 2014.

25. Miani D, Nucifora G, Piccoli G, Proclemer A and Badano LP: Incremental value of three-dimensional strain imaging in danon disease. Eur Heart J Cardiovasc Imaging 13: 804, 2012.

26. Piotrowska-Kownacka D, Kownacki L, Kuch M, Walczak E, Kosieradzka A, Fidzianska A and Krolicki L: Cardiovascular magnetic resonance findings in a case of danon disease. J Cardiovasc Magn Reson 11: 12, 2009.

27. Le DD, Alvarez P, Barrios R, Arriaga M, Cherry M, Shah D and Lin $\mathrm{CH}$ : Hypertrophic cardiomyopathy with unusual extensive scarring pattern: Danon disease. Methodist DeBakey Cardiovasc J 12: 227-229, 2016.

28. Kitahara H, Nawata K, Kinoshita O, Itoda Y, Shintani Y,Fukayama M and Ono M. Implantation of a left ventricular assist device for danon cardiomyopathy. Ann Thorac Surg 103: e39-e41, 2017.

29. Zaki A, Zaidi A, Newman WG and Garratt CJ: Advantages of a subcutaneous implantable cardioverter-defibrillator in LAMP2 hypertrophic cardiomyopathy. J Cardiovasc Electrophysiol 24: 1051-1053, 2013.

\footnotetext{
This work is licensed under a Creative Commons Attribution-NonCommercial-NoDerivatives 4.0 International (CC BY-NC-ND 4.0) License.
} 